\title{
Las nuevas clases medias en Chile: Un análisis de cohortes
}

\author{
Oscar Mac-Clure
}

En este artículo se utiliza la metodología del análisis de cohortes para estudiar a las actuales "nuevas" clases medias chilenas, describiendo su composición y comparándolas con las cohortes previas que continúan en actividad laboral. Entendidas las clases medias como estratos definidos según la ocupación, se analiza el tamaño relativo de las cohortes que las componen durante las décadas de 1990 y 2000, distinguiendo entre "nuevas" y "viejas" cohortes. Se examina si el surgimiento de nuevas cohortes de acuerdo con sus ocupaciones guarda también correspondencia con diferencias en los ingresos. Se concluye que ha surgido una nueva clase media entre quienes se incorporaron plenamente a la actividad laboral en el período de crecimiento económico de las décadas de 1990 y 2000. En el interior de las nuevas clases medias, su estrato superior ocupa un lugar preeminente y la pertenencia de clase influye en los ingresos de las personas. 


\section{I}

\section{Introducción}

Las clases medias han suscitado creciente interés en América Latina debido a la inquietud provocada por la persistencia de desigualdades sociales más allá de la pobreza, pero en parte también porque representan referencias simbólicas que aparecen como una promesa para amplios sectores de la población que han accedido a la expansión del consumo (Franco, Hopenhayn y León, 2010). En el plano del poder político, en América Latina y en Chile, la matriz sociopolítica de gran parte del siglo XX ha sido reemplazada por una autonomía relativa de la economía respecto de la política, dificultando el surgimiento de categorías sociales - como la clase obrera en el pasado histórico- que aglutinen a grandes conglomerados de la población frente a las estructuras predominantes, de acuerdo con Garretón (2007). Sin embargo, es posible que el panorama social, cultural y político se esté viendo alterado por el surgimiento de "nuevas" clases medias, un cambio que involucra sobre todo a la generación más reciente, según se expone en este artículo.

A nivel mundial, incluida la región latinoamericana, se ha afirmado que en las últimas décadas, como corolario de la disminución de la pobreza, ha surgido una clase media "emergente", pero todavía vulnerable al riesgo de volver a descender en la escala de ingresos (Banerjee y Duflo, 2008; Kreckel, 2006; Ravallion, 2009; OCDE, 2010). Resulta de interés estudiar esta clase media emergente en un caso específico como el de Chile, pero hay que agregar que debido a la rapidez del cambio ocurrido, el proceso afecta especialmente a la población más joven, dando forma a una nueva generación de clase media. Además, se ha enfatizado en estudiar la movilidad ascendente desde los estratos más bajos hacia las posiciones medias, pero se ha prestado

\footnotetext{
$\square$ La variable clases sociales (EGP) para las encuestas aplicadas en Chile fue elaborada por Vicente Espinoza, a quien se agradece sinceramente su apoyo, como también a José Pujol, por su contribución al análisis de cohortes, a Álvaro Krause, por su apoyo al cálculo de los índices de Gini y a Víctor Maturana, por su colaboración en el modelo de regresión aplicado. Asimismo, se agradecen los valiosos comentarios de Emmanuelle Barozet, Gonzalo Delamaza, Vicente Espinoza, Arturo León y Javier Núñez. Este artículo se enmarca en el Proyecto Anillo SOC12, inserto en el Proyecto Desigualdades y financiado por la Comisión Nacional de Investigación Científica y Tecnológica (Conicyt).
}

menos atención a qué estaría ocurriendo en el resto de las clases medias, particularmente en sus estratos superiores, por lo que en este artículo se examina no solo un sector, sino el conjunto de los integrantes de estas clases intermedias.

Siguiendo a Bourdieu (1987), las posiciones medias pueden caracterizarse por su indeterminación económica y cultural —su carácter "intermedio"- en un determinado momento o período, pero también se desarrollan a lo largo del tiempo. El porvenir que una posición media ofrece a quienes se sitúan en ella está relativamente predeterminado, prometiendo con una relativa certeza un ascenso o descenso. Esto permite distinguir posiciones medias descendentes en comparación con otras posiciones con un futuro estable o ascendente. De acuerdo con este autor, en las posiciones medias que prometen un futuro más seguro es donde con mayor intensidad actúan sus integrantes en búsqueda de un ascenso, lo que configura a una "nueva" clase media. Poniendo atención al horizonte o devenir de las nuevas clases medias, más que a lo que ocurre en un momento o período dados, Bourdieu asigna especial relevancia a las particularidades generacionales. Desde esta perspectiva, en el presente artículo se presta atención tanto a la identificación de sectores en ascenso como, por el contrario, a la presencia de sectores en declinación en las nuevas generaciones de clases medias.

El término "generación" al que alude aquí la visión de la nueva clase media, se remonta en el campo de la sociología a la teoría de Mannheim (1952), quien sostuvo que si los individuos comparten una misma etapa en el ciclo vital, como el año de nacimiento, se encontrarán en una situación parecida a la de otros frente a la dinámica del acontecer social, como los cambios en las condiciones económico-sociales. Esta pertenencia a una misma generación establece un modo de insertarse en diversos ámbitos de la sociedad. A modo de ilustración, las personas nacidas en un mismo año, que se incorporan al mundo laboral en un período similar y con un semejante nivel de educación, comparten opciones relativamente delimitadas y diferentes a quienes no tienen esas características.

Un concepto más específicamente definido, el de "cohorte" (Ryder, 1965; Glenn 2005), permite observar a los individuos que comparten una característica inicial, 
como el año de nacimiento, efectuando un seguimiento del grupo mediante datos cuantitativos para observar la incidencia de uno o más eventos a lo largo del tiempo ${ }^{1}$. El concepto de cohortes ha sido aplicado en estudios sobre clases medias en otros países (Chauvel, 2002) y se utiliza en este artículo proponiendo un nuevo método para el análisis cuantitativo de datos de la principal serie de encuestas de hogares del país, las encuestas de Caracterización Socioeconómica Nacional (CASEN) de 1990 a 2009 (Ministerio de Planificación, actualmente Ministerio de Desarrollo Social). Estudiar las "nuevas" cohortes que componen la clase media, que todavía tienen un tramo prolongado por recorrer en su ciclo de vida a diferencia de la "viejas" cohortes, puede aportar aquello no ofrecido por metodologías en que se utilizan datos sobre la población en general: un análisis sistemático relacionado con una posible trayectoria actual y futura de esta clase. En un sentido más amplio, estudiar las clases medias chilenas desde la perspectiva de las cohortes que la integran puede contribuir a un mayor conocimiento de estas clases, así como a entender mejor las desigualdades y los procesos sociales.

${ }^{1}$ El método de análisis de cohortes fue desarrollado inicialmente en estudios demográficos y es actualmente de amplio uso en campos como la medicina y la educación.
En el caso de Chile, la liberalización de los mercados y la expansión de las ocupaciones en el sector terciario, tal como la ampliación de la educación, han influido en la composición de las clases sociales y las oportunidades de movilidad social (León y Martínez, 2001; Torche y Wormald, 2004). A este proceso gradual se agregó el paso de un período de estancamiento e inestabilidad económica a uno de crecimiento relativamente elevado en las décadas de 1990 y 2000. La hipótesis de este trabajo es que esto habría ejercido un efecto diverso entre las cohortes que iniciaron una trayectoria laboral específicamente en estas dos décadas, en comparación con quienes tenían una inserción ocupacional establecida desde antes de este período de particulares características. Se sostiene aquí que los cambios en el empleo y los ingresos generados por el crecimiento económico durante las décadas antes mencionadas, no fueron experimentados de modo similar entre quienes integraron las clases medias, sino más intensamente por sus cohortes más recientes que se incorporaron de lleno a la actividad laboral en este período.

Luego de esta Introducción, el artículo se desarrolla de la siguiente manera. En la sección II se aborda el tránsito de la clase media a las clases medias. La sección III se refiere a las cohortes de clases medias en un período de crecimiento económico. En la sección IV las cohortes de clases medias son vistas en un contexto de desigualdad de ingresos y "meritocracia". Por último, en la sección V se entregan las conclusiones.

\section{II}

\section{De la clase media a las clases medias}

Para enfocar a la clase media, reconociendo su composición heterogénea, se requiere estudiar las clases medias. Una forma de definir a las clases medias y observar esas distinciones consiste en combinar dimensiones como la ocupación, los ingresos y los bienes de consumo disponibles en el hogar, lo que ha permitido una descripción de estas clases en Chile y otros países latinoamericanos (Franco, Hopenhayn y León, 2011). Para el análisis de cohortes, en este artículo se enfatizan las diferencias entre los integrantes de las clases medias respecto del trabajo que desempeñan, que son centrales para sus ingresos, sus modos de vida e identidades culturales.

Desde esa perspectiva ocupacional, la composición de la estructura de clases puede ser estudiada a partir de diversas clasificaciones ${ }^{2}$. Entre estas, ante todo para mantener estándares de "comparabilidad", aquí se utiliza la de Erikson y Goldthorpe (1993), una de las más aplicadas a nivel internacional y en los estudios recientes más relevantes en Chile (Torche y Wormald, 2004; Espinoza y Barozet, 2009). Se trata de una tipología basada en ocupaciones, en que se distinguen categorías a partir de la combinación de tres criterios: propiedad y control de los medios productivos, prestación de servicios con mayor o menor autonomía, y manualidad con mayor o menor grado de calificación. Las ocupaciones

\footnotetext{
${ }^{2}$ Con ese enfoque, para un completo examen de las categorizaciones más frecuentemente utilizadas, véase Leiulfsrud, Bison y Jensberg (2005).
} 
específicas son clasificadas en una u otra clase social definida según esos criterios, combinando variables disponibles en los datos estadísticos sobre ocupados: categoría ocupacional, actividad económica, oficio y tamaño de empresa.

Los grandes y medianos empresarios representaron un $1,2 \%$ del total de ocupados en el año 2009, pero se excluirán del análisis considerando que respecto de ellos se requeriría medir sus ingresos provenientes del capital, lo que no se obtiene con precisión de encuestas de hogares aplicadas en Chile, como las que se utilizarán en este caso ${ }^{3}$. Con esta delimitación, la composición de las clases sociales en el país de acuerdo con la clasificación antes indicada puede apreciarse en el gráfico 1, en que se muestra el porcentaje de ocupados por clase en el año 2009.

Siguiendo un criterio convencional en estudios sobre el tema, se considera que las clases medias están integradas por los primeros cuatro estratos de trabajadores no-manuales en el esquema de clasificación expuesto en el gráfico 1, abarcando el 54\% de las personas ocupadas en el país.

\footnotetext{
${ }^{3}$ En este trabajo se entiende por empresarios grandes o medianos a quienes declaran ser empleador o patrón en establecimientos de 10 o más trabajadores, de acuerdo con la encuesta CASEN del año 2009.
}

La denominada clase de servicios es el primer conjunto de ocupaciones que integran las clases medias. Está formada predominantemente por profesionales y técnicos de nivel alto contratados tanto en el sector privado como en el público, ejecutivos de empresas y funcionarios con cargos directivos en el Estado. Un escalón más abajo está integrada también en una elevada proporción por profesores, profesionales de servicios administrativos, técnicos con formación superior, profesionales de nivel medio de la salud y profesionales de la comunicación. En su mayor parte, estas personas cuentan con educación superior y se desempeñan mayoritariamente en actividades económicas del sector servicios, aunque también en otras ramas.

El segundo componente de las clases medias es la denominada clase de rutinas no-manuales, cuyos empleos más frecuentes corresponden en su estrato alto a personas contratadas como vendedores de tiendas y empleados administrativos, a los que —en un estrato más bajose agregan secretarias y cajeros. Se trata de asalariados que en su mayoría solo cuentan con educación media y muy pocos tienen educación universitaria completa.

Se considera también como parte de las clases medias al estrato de independientes o trabajadores por cuenta propia no profesionales ni técnicos, principalmente vendedores de quioscos, puestos de mercado y almacenes, taxistas, carpinteros, costureras, peluqueros,

GRÁFICO 1
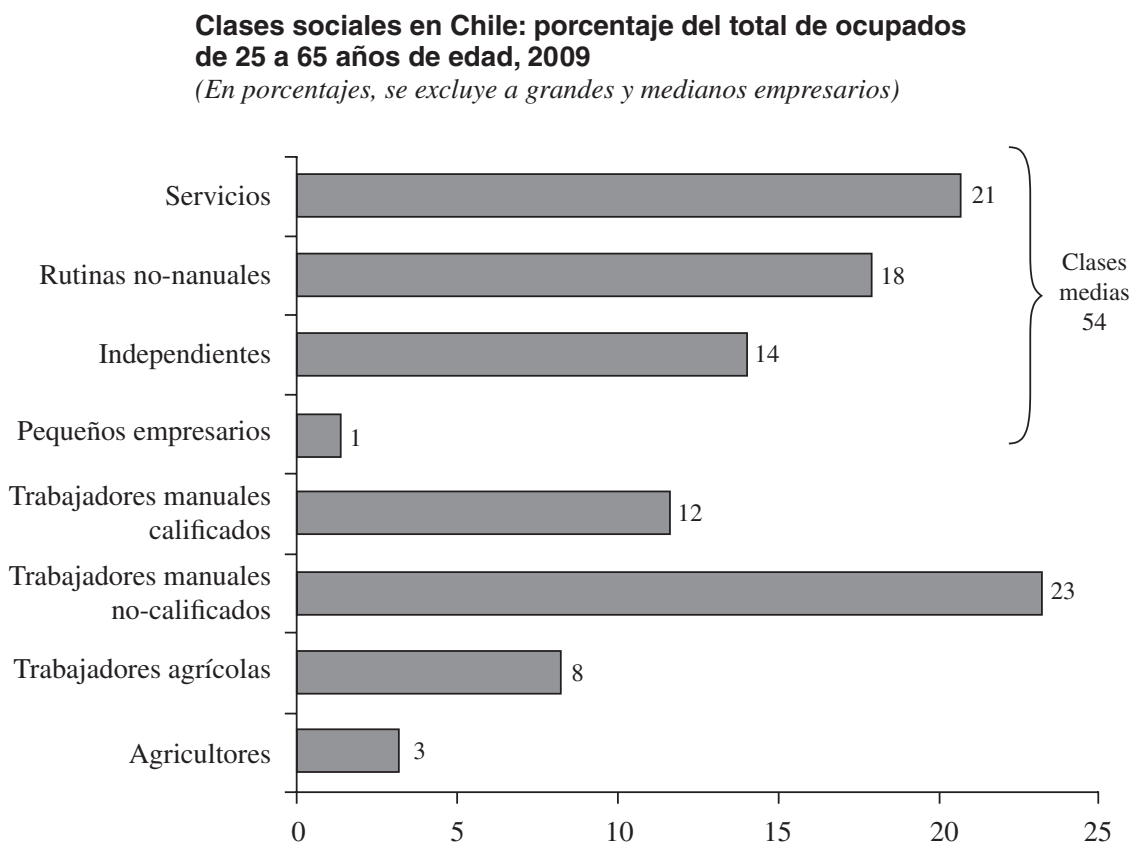

Fuente: Encuesta de Caracterización Socioeconómica Nacional (CASEN) de 2009. 
mecánicos de vehículos, artesanos de la madera y de técnicas tradicionales, entre otros. Se desempeñan en ocupaciones que requieren más experiencia que educación formal: alrededor de la mitad cuenta al menos con educación media, pero el resto no ha alcanzado ese nivel educacional. Una gran parte de ellos integra el sector "informal", si a modo de indicador se considera que la mayoría no cotizan en el sistema previsional. Tomando en cuenta sus actividades independientes o no asalariadas como rasgo distintivo para incluirlos en las clases medias, son asimilables a la categoría que históricamente se denominó "pequeña burguesía", en conjunto con los pequeños empresarios. A estos últimos se los incluye como un cuarto estrato de las clases medias, identificando como pequeños empresarios a quienes emplean entre 2 y 9 trabajadores, un estrato comparativamente poco numeroso ${ }^{4}$.

Manteniendo exclusivamente el criterio ocupacional para establecer distinciones, otras categorías fuera de las clases medias corresponden a los estratos de trabajadores manuales, incluidos los calificados

\footnotetext{
4 Esta delimitación del número de asalariados —naturalmente arbitraria- corresponde a lo definido internacionalmente para la escala de estratificación EGP, del mismo modo que el resto de las definiciones aplicadas en nuestra clasificación. Sin embargo, esta cantidad de ocupados en la empresa corresponde a la forma como normalmente se clasifica a los pequeños empleadores con datos de las encuestas CASEN utilizadas.
}

y no-calificados. Son asalariados que desempeñan actividades manuales, lo que no corresponde a una definición convencional de clase media, aunque muchas de las personas integrantes de estas categorías pueden autoidentificarse o ser identificadas como de estrato medio más que de clase "baja". Entre los asalariados del estrato de trabajadores manuales calificados, los oficios más frecuentes corresponden a mecánicos, cocineros y soldadores, mientras que en los asalariados no-calificados se incluyen principalmente trabajadores menos calificados de la construcción, conductores de camiones, guardias de seguridad, personal de aseo de oficina y trabajadoras de servicio doméstico. Los trabajadores manuales no-calificados constituyen la clase más numerosa en el país y aunque no son incluidos como parte de las clases medias, deben ser incorporados en un análisis de estas como se verá más adelante.

A esto se agregan, finalmente, las clases de trabajadores agrícolas y agricultores, a quienes por sus actividades económicas -independientemente de su identidad subjetiva como clase baja, media o alta- se les considera alejados de una definición de las clases medias como vinculadas a las áreas urbanas.

Se sabe que la expansión de las actividades económicas de servicios y comercio ha generado un aumento de ocupaciones en estratos de clase media como los de servicios y de rutinas no-manuales, pero se desconoce en qué medida esto ha ocurrido en las cohortes más recientes o en las anteriores, lo que se examinará a continuación.

\section{III \\ Cohortes de clases medias en un período de crecimiento económico}

A las clases medias descritas de acuerdo con el esquema anterior, se aplica seguidamente la herramienta del análisis de cohortes para distinguir entre "nuevas" y "viejas" cohortes. Conocer la evolución de las nuevas cohortes de clases medias en el período de crecimiento económico de las décadas de 1990 y 2000 puede aportar una renovada e interesante perspectiva, como se verá enseguida.

En este análisis se parte del supuesto de que las variaciones en la participación de una u otra cohorte en la ocupación total obedecen a cambios estructurales que inciden en las clases sociales. De acuerdo con Bourdieu
(1987, pág. 350), las posiciones que experimentan un descenso cuantitativo expresan generalmente una decadencia económica y social. Es decir, se encuentran ligadas a un pasado acabado o en vías de extinción, mientras que a la inversa, las que aumentan son aquellas que representan el porvenir. Así, en el pasado histórico, Weber (1964, págs. 244 y 245) observó que el tránsito a la "pequeña burguesía independiente" de los artesanos había sido un "ideal" para los trabajadores manuales, pero esto dejó de ser una oportunidad relevante cuando se expandieron las clases medias integradas por empleados de oficinas comerciales y burócratas. 
Para abordar esa dinámica con respecto al caso de Chile en la actualidad, se analizarán cohortes de clases medias según su año de nacimiento, utilizando la serie de encuestas de hogares CASEN llevadas a cabo durante el período de crecimiento económico que nos interesa; estas permiten examinar la evolución de esas cohortes en el transcurso de las décadas de 1990 y $2000^{5}$.

En el análisis de cohortes se utiliza el clásico diagrama de Lexis, partiendo del principio de que si se dispone de encuestas representativas de la población, como la serie indicada, las personas que tenían una cierta edad cuando se aplicó la primera encuesta en 1992, tendrán 17 años más en la última encuesta del año 2009, es decir, forman parte de una misma cohorte ${ }^{6}$. A modo de ejemplo, quienes tenían 25 años de edad en 1992 habían nacido en 1967 y tenían 42 años de edad en la encuesta efectuada en 2009, asumiendo como supuesto que las personas encuestadas en cada oportunidad son representativas globalmente de los individuos nacidos en el mismo año, es decir, integran una misma cohorte ficticia de acuerdo con el supuesto indicado. Lo mismo se aplica para todas las encuestas, lo que difiere del método habitual de constituir grupos etarios en concordancia con rangos de las mismas edades en una serie de encuestas.

En los gráficos siguientes se analizan los resultados obtenidos. El porcentaje corresponde a los pertenecientes a una clase en el total de ocupados de la misma edad, cuando fueron encuestados. Las cohortes de clases medias son definidas según el año de nacimiento y cada línea graficada representa una cohorte. Para simplificar el análisis y atenuar errores de declaración, en este ejercicio se ha calculado un porcentaje correspondiente a grupos quinquenales de edad y trienales en el caso de las personas de menor y mayor edad: 25 a 27 años y 63 a 65 años, respectivamente. Así por ejemplo, cada punto graficado frente a la edad de 30 años corresponde al porcentaje de ocupados entre las edades de 28 a 32 años. Se considera a las personas ocupadas de 25 a 65 años de edad en el momento de aplicarse cada una de las encuestas CASEN.

\footnotetext{
5 Las encuestas CASEN utilizadas en este análisis de cohortes fueron aplicadas en los años 1992, 1994, 1998, 2000, 2003, 2006 y 2009.

${ }^{6}$ El diagrama de Lexis permite representar la dinámica de una población. Combina en un diagrama bidimensional dos dimensiones temporales, la dimensión del tiempo de calendario con la dimensión de la edad de los individuos. Esta combinación da lugar a una tercera dimensión temporal, la de una cohorte o una línea de vida, según el momento de nacimiento u otro evento relevante.
}

Los resultados de este método de análisis se aprecian en el gráfico 2, específicamente para la categoría superior de las clases medias, la clase de servicios?

En la clase de servicios el efecto de cohortes es claramente visible, según se constata en el gráfico 2. Las cohortes más recientes, particularmente las de 1970 a 1980, pertenecen en mayor proporción a este estrato superior de la clase media, en comparación con las cohortes anteriores. A una misma edad, por ejemplo a los 30 años de edad, se observa que la proporción de quienes se integran a la clase de servicios con respecto al total de ocupados en el país de la misma edad, ha experimentado una expansión en la cohorte de 1970 en comparación con la de 1965. Este cambio o efecto de cohortes no es muy notorio a partir de los 40 años de edad, pero se observa una diferencia en las cohortes más antiguas.

En contraste con la anterior, en la clase de independientes se observa una progresión por edad diferente, al mismo tiempo que una distinta participación de las nuevas cohortes en la ocupación total, de acuerdo con el gráfico 3.

A diferencia de la clase de servicios, a medida que aumenta la edad se incrementa el desarrollo de actividades independientes, aunque en menor proporción en las

${ }^{7}$ Agregamos los estratos alto y bajo de la clase de servicios debido a que los resultados son muy similares.

GRÁFICO 2

\section{Clase de servicios: porcentaje en los ocupados por año de edad, según cohortes, 1992-2009}

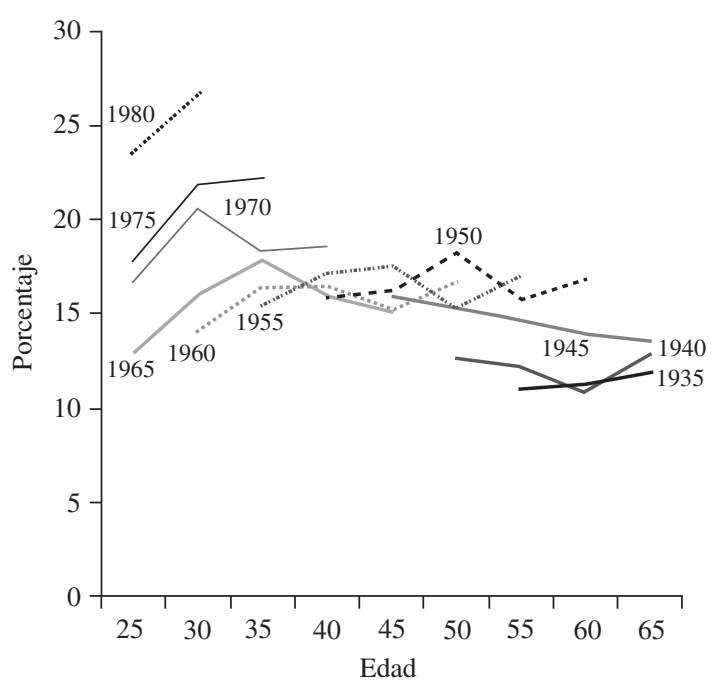

Fuente: Encuesta de Caracterización Socioeconómica Nacional (CASEN), 1992 a 2009. 
GRÁFICO 3

Clase de independientes: porcentaje en los ocupados por años de edad, según cohortes, 1992-2009

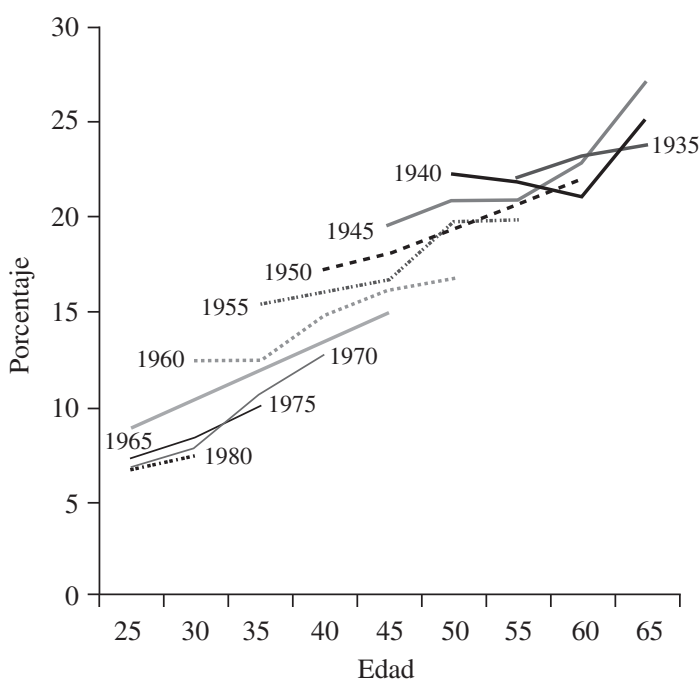

Fuente: Encuesta de Caracterización Socioeconómica Nacional (CASEN), 1992 a 2009.

cohortes más recientes que corresponden a los nacidos a partir de alrededor de 1970, según el gráfico 3. Resulta sobre todo interesante observar que contrariamente a la clase de servicios (véase el gráfico 2) cuyas nuevas cohortes se expanden a una misma edad, las mismas cohortes recientes, pero en la clase de independientes (véase el gráfico 3), experimentan una marcada disminución.

De modo inverso a la clase de trabajadores independientes, en la clase de rutinas no-manuales se aprecia una progresión por edad descendente y homogénea entre las cohortes, de acuerdo con el gráfico $4^{8}$.

A medida que aumenta la edad de las personas encuestadas, disminuye la proporción de quienes realizan trabajos que corresponden a la clase de rutinas no-manuales y esto ocurre de modo relativamente homogéneo en las diversas cohortes, incluidas las más recientes, según se observa en el gráfico 4 . En las nuevas cohortes, el efecto de la edad es más importante que el efecto de la cohorte.

De este modo, a diferencia de la clase de servicios, en la clase de rutinas no-manuales se mantiene el porcentaje de las nuevas cohortes, sin disminuir como en la clase de independientes. Una transformación en la

\footnotetext{
${ }^{8}$ Los estratos alto y bajo de la clase de rutinas no-manuales han evolucionado de modo similar.
}
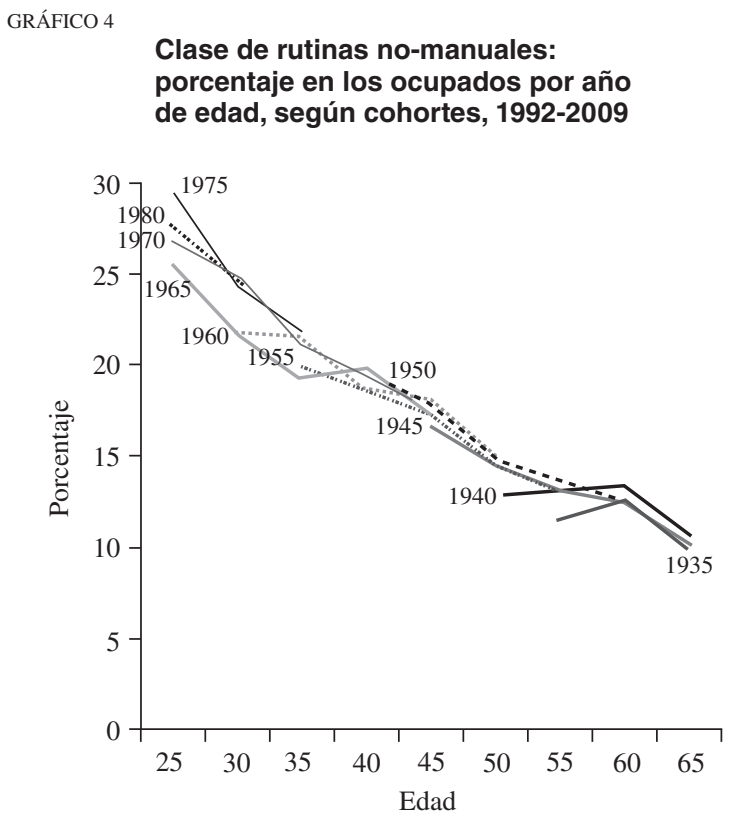

Fuente: Encuesta de Caracterización Socioeconómica Nacional (CASEN), 1992 a 2009.

composición de las clases medias es observable particularmente en la clase de servicios, que se expande en las cohortes correspondientes a las personas nacidas entre 1970 y 1984, de acuerdo con los datos quinquenales que se han expuesto. Considerando datos anuales, la línea de inflexión en la clase de servicios se sitúa más marcadamente en 1971.

Desde el punto de vista estricto de las diferencias cuantitativas, el aumento del tamaño del estrato de servicios en las nuevas cohortes se debe en parte a la declinación de la ocupación de estas cohortes en la clase de independientes, a lo que se agrega una declinación menor del empleo en la clase de trabajadores manuales no-calificados de las mismas cohortes.

Desde una perspectiva de la movilidad social intergeneracional, esta transferencia podría explicarse como una transición desde los padres a los hijos. Por una parte, un "ascenso" a la clase de servicios y, por otra, una "emergencia" desde fuera de las clases medias hacia la clase de rutinas no-manuales. En las nuevas cohortes de la clase de rutinas no-manuales, un $60 \%$ ha tenido padres pertenecientes a la clase de trabajadores manuales. En contraste, en las cohortes recientes de la clase de servicios, un 59\% de los padres ya han pertenecido a esta misma clase $u$ otro estrato de las clases medias y el resto a la clase de trabajadores manuales o 
agricultores 9 . Una parte de las nuevas cohortes de clase media consiste entonces en un sector "emergente" que busca mejores alternativas que la clase de trabajadores manuales a que pertenecían sus padres y logra insertarse en el estrato de rutinas no-manuales de las clases medias, pero a la vez un sector que denominaremos "ascendente" aprovecha la oportunidad de evitar esta última clase y accede a la clase de servicios. La clase de rutinas no-manuales permanece sin crecer en las nuevas cohortes, como resultado de que el sector "ascendente" logra distinguirse de este estrato e insertarse en la clase de servicios. Además, integrantes de las nuevas cohortes se alejan de la clase de independientes a la que pertenecieron sus padres, para pasar a formar parte ya sea del sector "emergente" o del "ascendente".

Los resultados de nuestro análisis de las cohortes de clase media permiten agregar dos importantes aspectos a una explicación intergeneracional como la reseñada. En primer lugar, la transición experimentada por un sector "ascendente" y uno "emergente" es inherente a las nuevas cohortes de clase media, pues los cambios que se han expuesto ocurren en estas cohortes con mayor intensidad que en las viejas cohortes durante las décadas de 1990 y 2000 . De este modo, contrariamente a lo que podría creerse, no se trata de un fenómeno generalizado en las clases medias. En segundo lugar, aunque las apariencias vinculadas a la expansión del consumo apunten a poner de relieve el surgimiento de una clase media "emergente" proveniente de las clases "bajas" y de una situación de pobreza o vulnerabilidad social, una transición involucra también a un sector "ascendente" dentro de las nuevas cohortes de clases medias, que evoluciona hacia el estrato superior de estas clases aumentando el tamaño de la clase de servicios según lo descrito previamente.

Cabe subrayar que los resultados de este trabajo se sitúan estrictamente en el período comprendido entre las décadas de 1990 y 2000, sin abarcar biografías familiares o procesos de larga duración y allí reside también su

\footnotetext{
${ }^{9}$ Porcentajes a partir del análisis de datos de la Encuesta Nacional de Estratificación Social, aplicada por el Proyecto Desigualdades (Anillo SOC12) a una muestra representativa a nivel nacional y regional en el año 2009. En el porcentaje de padres trabajadores manuales se incluyen trabajadores agrícolas.
}

potencialidad para contribuir a explicar la evolución de largo plazo. Como se ha visto, si se considera el criterio de quienes experimentan un incremento en su participación en el empleo durante el período estudiado, los resultados obtenidos permiten afirmar que las nuevas cohortes de clases medias están representadas ante todo por las cohortes de quienes nacieron a partir de $1970 \mathrm{y}$ pertenecen a la clase de servicios, que desempeña un papel motriz. Esto incide en la dinámica interna del estrato de rutinas no-manuales en las clases medias y, de modo más amplio, afecta al conjunto de las nuevas cohortes de clases medias en el período reciente.

De acuerdo con los resultados obtenidos en el presente análisis, las cohortes de quienes nacieron a partir de 1970 tienen una pertenencia de clases diferente a las cohortes anteriores. Esta inflexión, definida por los que nacieron a partir de 1970, afecta a quienes cumplieron 25 años desde 1995. Esto implica que ingresaron plenamente al mercado laboral durante el período de crecimiento económico de las décadas de 1990 y 2000, un aspecto definitorio de las nuevas cohortes de clases medias. El crecimiento económico intensificó transformaciones en la estructura del empleo, debido a su vez a cambios en la gravitación de las distintas actividades económicas. En todas las economías latinoamericanas se ha registrado un incremento del sector terciario, junto con una pérdida de importancia de los sectores industrial y agrícola. Lo que agrega este análisis de cohortes de las clases medias es que esto se manifiesta más pronunciadamente y sobre todo en las nuevas cohortes que en las viejas cohortes.

Lo anterior no constituye un asunto menor desde el punto de vista del tamaño de las nuevas cohortes, pues en las clases medias los nacidos a partir de 1970 pasaron de ser un 7\% de estas clases en 1996 a un 44\% en 2009.

En los resultados obtenidos se advierte una nueva composición de las clases medias en el período reciente desde un punto de vista de cohortes. En este marco, la siguiente pregunta se refiere a si solo se trata de un cambio en la composición de las clases medias o bien, siguiendo la corriente clásica en el estudio de las clases sociales, desde autores como Marx y Engels (1989) y Weber (1964), lo importante ante todo es si esto conlleva desigualdades de acceso a los recursos económicos y a la distribución de los ingresos. 


\section{IV \\ Cohortes de clases medias, desigualdad de ingresos y "meritocracia"}

Para corroborar si lo expuesto hasta aquí es algo más que un cambio ocupacional, se revisará si la pertenencia a las nuevas cohortes guarda correspondencia con diferencias en los ingresos respecto de las viejas cohortes. Se reexaminará si la desigualdad aumenta o disminuye en las nuevas cohortes y cuáles son los factores que influyen en sus ingresos.

Para apreciar la desigualdad de ingresos, el indicador con mayor aceptación en los trabajos empíricos es el coeficiente de Gini. Con los datos de que se dispone es posible examinar este indicador en el conjunto de los ocupados, para establecer si la desigualdad es mayor o menor en los dos grupos de cohortes que se han revisado previamente de acuerdo con las edades de sus integrantes, sin pretender una mayor desagregación, pues ello entrañaría problemas de representatividad estadística. La distribución del coeficiente de Gini para los ingresos autónomos individuales de los ocupados según su edad, respecto de las nuevas y viejas cohortes, se presenta en el gráfico $5^{10}$. Como las nuevas cohortes que se han identificado abarcan a quienes nacieron a partir de 1970, esto incluye a los ocupados de hasta 39 años de edad en la encuesta del año 2009, lo que define el límite superior del rango etario que resulta pertinente comparar.

La desigualdad de ingresos según el coeficiente de Gini es, en general, relativamente baja en las personas más jóvenes y aumenta con la edad, aunque tiende a estabilizarse en las nuevas cohortes de acuerdo con el gráfico 5. Además, el coeficiente de Gini en las nuevas cohortes es inferior en comparación con las viejas cohortes - una diferencia promedio de 0,04 puntos del índice-, lo que es consistente con lo analizado por Sapelli (2011a) respecto del conjunto de la población chilena. Es decir, se observa una alteración de la tendencia

\footnotetext{
${ }^{10}$ Los problemas de representatividad estadística —que limitan el efectuar desagregaciones - surgen del hecho de que como primer paso se requiere calcular un coeficiente de Gini para los individuos de cada año de edad en cada una de las encuestas. Con el objeto de atenuar el error estadístico que esto ocasiona, en todas las encuestas de este trabajo se utilizan grupos móviles de cinco años para el cálculo de los coeficientes de Gini correspondientes a cada año de edad. Las nuevas y viejas cohortes corresponden a promedios simples de los coeficientes de Gini de las respectivas cohortes anuales.
}

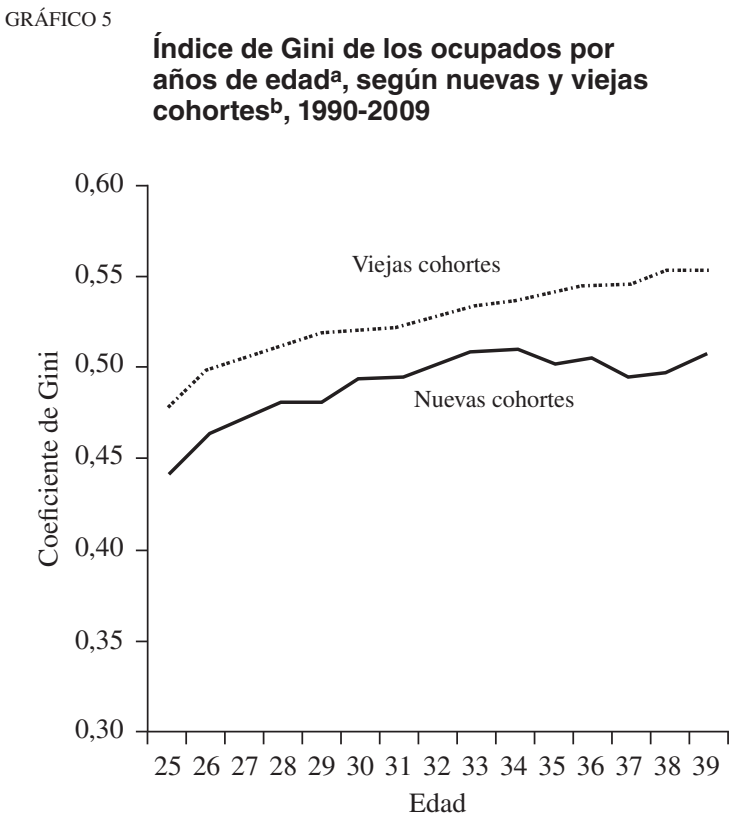

Fuente: Encuesta de Caracterización Socioeconómica Nacional (CASEN), 1992 a 2009.

Nota: el ingreso autónomo personal corresponde a la variable YAUTAJ en la Encuesta CASEN.

a Coeficiente de Gini calculado en promedios móviles de años sobre el ingreso autónomo personal para personas de 25 a 39 años.

b Nuevas cohortes: nacidos a partir de 1970.

Viejas cohortes: nacidos antes de 1970.

general en las cohortes más recientes, que experimentan una menor desigualdad a una misma edad en comparación con las cohortes anteriores. Por ejemplo, a los 35 años de edad el coeficiente de Gini es de 0,53 entre los ocupados pertenecientes a la cohorte de quienes nacieron antes de 1970, mientras que de solo 0,50 en la cohorte de los nacidos a partir de ese año, durante el período comprendido entre 1990 y 2009. En este ejercicio se ha calculado el coeficiente de Gini promedio durante el período analizado respecto de los dos grandes grupos de cohortes que se han distinguido empíricamente, el correspondiente a los nacidos antes de 1970 y el de los nacidos de este año en adelante, pero si se analizan los 
índices de los nacidos específicamente en cada año, el salto más notorio ocurre entre quienes nacieron en 1971.

El menor coeficiente de Gini en todas las edades en las nuevas cohortes, al comparar con las viejas cohortes según el gráfico 5, corrobora la distinción anterior entre ambos grupos de cohortes, ya que muestra un nítido efecto de las cohortes y no solo de las edades de las personas. Esta confirmación se refiere al conjunto de los ocupados, aunque no es posible especificarla en las clases medias debido a los problemas de representatividad estadística ya mencionados, pero más adelante esto se retomará examinando la incidencia en los ingresos de la pertenencia a cada uno de los estratos de clase media, tanto en las nuevas como en las viejas cohortes.

Una explicación de la menor desigualdad de ingresos en las nuevas cohortes abre dos tipos de discusión respecto de las clases medias. En primer lugar, desde un punto de vista intercohorte, cabe preguntarse si esa menor desigualdad puede atribuirse al menos en parte al ascenso del empleo en el estrato de servicios en estas cohortes, integrado predominantemente por personas que han completado estudios universitarios o técnicos. Esto en principio introduce un efecto "meritocrático" e igualador —lo que se revisará más adelante—, especialmente al extenderse ese tipo de educación más allá de una élite, proceso que viene desarrollándose gradualmente en Chile desde hace varias décadas. En segundo lugar, dentro de las nuevas cohortes de clases medias surge la pregunta sobre qué factores ejercen mayor incidencia en la menor desigualdad observada.

La evolución del coeficiente de Gini según cohortes en el período reciente, de acuerdo con el gráfico 5, también plantea un problema general desde el punto de vista del nivel de la desigualdad, pues en las nuevas cohortes el coeficiente continúa siendo de todos modos bastante elevado, lejos por ejemplo del índice de Gini promedio de 0,31 de los países de la OCDE (OCDE, 2011). Así, la desigualdad de ingresos en las nuevas cohortes dista de ser satisfactoria, como se ha sostenido (Sapelli, 2011b). El mayor tamaño de la clase de servicios en las nuevas cohortes no se traduce en una disminución sustancial de la desigualdad, lo que abre una discusión sobre la incidencia de la educación como factor igualador y, de modo aún más general, acerca de cuáles son las causas de la persistencia de niveles tan elevados de desigualdad en ambos grupos de cohortes.

Sin pretender establecer directamente una relación causal entre la evolución del coeficiente de Gini en el conjunto de la sociedad chilena y las clases medias, lo expuesto abre líneas de discusión y pone de relieve el interés de analizar con mayor atención la desigualdad en las nuevas cohortes de clases medias, como se hará a continuación.

Manteniendo la definición de las nuevas cohortes como los nacidos a partir de 1970, enseguida se analizarán empíricamente las diferencias de ingresos en las nuevas y viejas cohortes de clases medias, observando qué factores influyen en esas desigualdades y en qué medida se basan en la pertenencia a estas clases. Para esto se utilizará un modelo de regresión lineal múltiple, similar al aplicado por Leiulfsrud, Bison y Jensberg (2005) respecto del conjunto de los ocupados en los países europeos. Esto permite controlar el efecto de variables con demostrada influencia en los ingresos, como la educación y la experiencia laboral cuya incidencia fue postulada originalmente por Mincer (1974) y Becker (1975).

Este modelo de regresión permite apreciar la relevancia de la pertenencia a una clase social respecto de los ingresos para quienes tienen un mismo sexo, iguales años de experiencia laboral y similar nivel de educación, sin considerar interacciones entre estas variables. Específicamente, los resultados presentados en el cuadro 1 tienen como referencia el ingreso autónomo promedio de un hombre que carece de educación básica completa e integra la clase de trabajadores manuales no-calificados, considerando - por una parte- a las personas pertenecientes a las viejas cohortes de quienes nacieron entre 1944 y 1969, mientras que por otra se abarca a quienes integran las nuevas cohortes de los nacidos entre 1970 y 198411 . Los resultados corresponden a cada una de las encuestas CASEN efectuadas durante el período de 1998 a 2009.

Los resultados expuestos en el cuadro 1 muestran de un modo general que tener educación superior es lo que ejerce en promedio mayor influencia en los ingresos, pero estos se explican no solo por el nivel de educación y los años de experiencia laboral, sino que también incide y significativamente la clase social de pertenencia. Esto constituye un aporte relevante de los resultados del análisis, porque entonces ser integrante de las clases medias también determina una parte sustancial del ingreso autónomo de una persona. Además, los ingresos autónomos de las clases medias presentan

\footnotetext{
${ }^{11}$ Se podría realizar un ejercicio similar aplicando la misma regresión lineal al conjunto de los datos sin separar entre cohortes, sino agregando una variable dicotómica (dummy) que distinga entre nuevas y viejas cohortes. Sin embargo, esto implicaría especificar en cada encuesta los grupos de edad correspondientes a dichas cohortes, una combinación o subconjunto no independiente de las variables de experiencia — así como datos de edad-, de modo que la elevada correlación esperable entre esas variables predictivas invalidaría los resultados acerca de cada variable.
} 


\begin{tabular}{|c|c|c|c|c|c|c|c|c|c|c|}
\hline & \multicolumn{5}{|c|}{ Nacidos entre 1944 y 1969} & \multicolumn{5}{|c|}{ Nacidos entre 1970 y 1984} \\
\hline & 1998 & 2000 & 2003 & 2006 & 2009 & 1998 & 2000 & 2003 & 2006 & 2009 \\
\hline Mujer & $-0,25$ & $-0,24$ & $-0,24$ & $-0,27$ & $-0,26$ & $-0,25$ & $-0,25$ & $-0,24$ & $-0,29$ & $-0,26$ \\
\hline Experiencia aparente (edad-años estudio-6) ${ }^{\mathrm{b}}$ & 0,34 & 0,28 & 0,29 & 0,26 & 0,21 & $-0,09$ & 0,08 & 0,13 & 0,18 & 0,15 \\
\hline Experiencia aparente al cuadrado & $-0,26$ & $-0,17$ & $-0,24$ & $-0,24$ & $-0,23$ & 0,01 & $-0,07$ & $-0,10$ & $-0,12$ & $-0,09$ \\
\hline Educación básica completa & 0,08 & 0,10 & 0,09 & 0,09 & 0,08 & 0,03 & 0,06 & 0,06 & 0,07 & 0,07 \\
\hline Educación media incompleta & 0,16 & 0,17 & 0,15 & 0,15 & 0,12 & 0,08 & 0,13 & 0,13 & 0,13 & 0,11 \\
\hline Educación media completa & 0,27 & 0,29 & 0,27 & 0,24 & 0,22 & 0,18 & 0,27 & 0,27 & 0,28 & 0,27 \\
\hline Educación técnica o universitaria incompleta & 0,18 & 0,16 & 0,16 & 0,14 & 0,12 & 0,13 & 0,16 & 0,19 & 0,19 & 0,17 \\
\hline Educación técnica o universitaria completa & 0,41 & 0,38 & 0,41 & 0,35 & 0,30 & 0,27 & 0,37 & 0,40 & 0,40 & 0,39 \\
\hline Servicios & 0,21 & 0,20 & 0,20 & 0,20 & 0,21 & 0,31 & 0,28 & 0,31 & 0,28 & 0,30 \\
\hline Rutinas no-manuales & 0,12 & 0,11 & 0,10 & 0,12 & 0,14 & 0,21 & 0,19 & 0,16 & 0,17 & 0,18 \\
\hline Pequeños propietarios & 0,19 & 0,17 & 0,19 & 0,17 & 0,16 & 0,14 & 0,11 & 0,15 & 0,13 & 0,12 \\
\hline Independientes & 0,26 & 0,21 & 0,23 & 0,23 & 0,27 & 0,25 & 0,19 & 0,21 & 0,19 & 0,22 \\
\hline Trabajadores manuales calificados & 0,09 & 0,11 & 0,10 & 0,13 & 0,13 & 0,14 & 0,13 & 0,15 & 0,16 & 0,16 \\
\hline Trabajadores agrícolas & 0,02 & 0,06 & 0,04 & 0,07 & 0,09 & 0,05 & 0,07 & 0,06 & 0,07 & 0,08 \\
\hline Agricultores & 0,12 & 0,10 & 0,14 & 0,17 & 0,17 & 0,10 & 0,07 & 0,10 & 0,11 & 0,12 \\
\hline
\end{tabular}

Fuente: Encuesta de Caracterización Socioeconómica Nacional (CASEN), 1992 a 2009.

Notas: La variable dependiente corresponde al logaritmo del ingreso autónomo individual. El ingreso autónomo personal corresponde a la variable YAUTAJ en la Encuesta CASEN.

Se excluye del análisis a grandes y medianos empresarios (empleadores o patrones de la clase de servicios). Los resultados son estadísticamente significativos al $1 \%$ para todas las variables en ambas cohortes y en todos los años, excepto las variables sobre experiencia laboral en las nuevas cohortes de la encuesta CASEN de 1998.

a Nuevas cohortes: nacidos a partir de 1970. Viejas cohortes: nacidos antes de 1970.

b La experiencia aparente se calcula como edad-años de estudio-6.

cierta similitud, pues son superiores a los ingresos de la clase de trabajadores manuales no-calificados. Sin embargo, los ingresos de las clases de rutinas no-manuales se hallan comparativamente más próximos a los de los trabajadores manuales no-calificados. Todas estas diferencias proporcionales se mantienen y las variables son estadísticamente significativas al aplicar la misma regresión lineal al conjunto de los ocupados de entre 25 y 65 años, sin distinguir entre cohortes.

Una comparación intercohortes entre las nuevas y viejas cohortes es especialmente importante para los fines de este estudio. De acuerdo con el cuadro 1, la pertenencia de clase tiene mayor incidencia relativa en los ingresos en las nuevas cohortes de las clases medias de servicios y rutinas no-manuales, que entre quienes integran las cohortes previas.

En el interior de las nuevas cohortes, la clase de servicios tiene un nivel de ingresos relativos categóricamente discontinuo con respecto a las restantes clases medias y más aún en relación con las clases de trabajadores manuales. Es decir, la situación ocupacional de quienes integran las nuevas cohortes de la clase de servicios, les otorga una capacidad de acceso a mayores ingresos.
Desde el punto de vista intracohorte, también es relevante la evolución experimentada sobre todo en las nuevas cohortes, pues indica no solo una trayectoria pasada sino una posible tendencia. Merece destacarse cómo han evolucionado la incidencia de contar con educación superior y el efecto de la pertenencia de clase en el caso de la clase de servicios, que como hemos visto, desempeña un papel central durante el período analizado. A este respecto, los resultados del cuadro 1 se visualizan en el gráfico 6 .

Como se observa en el gráfico 6, en las nuevas cohortes, el efecto en los ingresos de contar con educación universitaria o técnica completa fue porcentualmente creciente hasta principios de la década de 2000, en comparación con las remuneraciones promedio de los trabajadores manuales no-calificados sin educación básica completa. Pero desde entonces ese efecto se ha estancado e incluso declinado, en paralelo a la ampliación de los egresados de universidades e institutos profesionales de formación técnica; es decir, se constata el conocido efecto de devaluación de la educación cuando esta se masifica. En cambio, la pertenencia a la clase de servicios mantiene su incidencia en los ingresos, aunque 
GRÁFICO 6

Efecto relativo en los ingresos de la educación superior completa y de la pertenencia a la clase de servicios en las nuevas cohortes, 1998-2009

(En porcentajes)

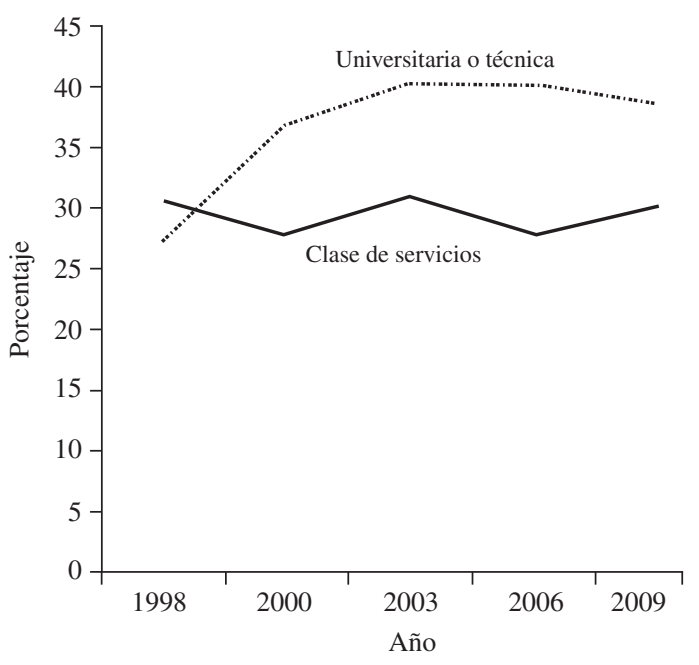

Fuente: sobre la base de datos del cuadro 1.

con altibajos. Estos resultados permiten medir el efecto de la educación superior completa, lo que se podría precisar considerando diferencias de calidad y prestigio entre una u otra universidad o instituto profesional, así como también accesos diferenciados a una educación de calidad según la clase de origen de los padres.

Como para las nuevas cohortes de la clase de servicios el mero título universitario se desvaloriza, adquiere importancia relativa en las remuneraciones de una persona el simple hecho de tener una ocupación de esa clase, según se desprende del gráfico 6. Esto contribuye a entender por qué la desigualdad de ingresos se mantiene elevada a pesar de la expansión de la educación superior, incluso en las nuevas cohortes de la población ocupada, según se advirtió previamente al examinar el índice de Gini de estas cohortes en el gráfico 5. Si se aspira a obtener una mejor posición en cuanto a los ingresos, además de un título se requiere tener contactos en la red de quienes pertenecen a la clase de servicios desde el punto de vista ocupacional. Para esta pertenencia de clase podrían incidir factores tales como haber podido acceder a una enseñanza escolar de mayor calidad y a una universidad prestigiada, las redes sociales de las que forman parte las personas —el "capital social" en un sentido más amplio (Barozet, 2006) - e incluso el hecho de compartir ciertos referentes culturales —un "capital cultural" de modo general. Además, se sostiene que la constante incorporación del cambio tecnológico, provocado por las nuevas tecnologías de la información y la comunicación (TIC) —aspecto relevante de la globalización—, es mayor entre quienes cuentan con educación superior, en contraste con los que desempeñan labores "rutinarias" que solo demandan educación media. Esto agudiza las desigualdades de ingresos (Autor, Katz, Krueger, 2008) por lo que cabría discutir y examinar en qué medida ello incide en la mantención de esas desigualdades en las nuevas cohortes, y si más allá de la educación formal inicial el proceso de absorción tecnológica se intensifica entre quienes ya se han insertado en ocupaciones de la clase de servicios.

Lo que aportan los resultados obtenidos consiste en que la diferenciación de ingresos basada en la pertenencia a clases sociales se encuentra vigente para la mayoría de quienes integran las nuevas cohortes de clases medias, incluso más que entre las viejas cohortes de estas clases intermedias - excepto los independientes y pequeños empresarios. Pertenecer a las nuevas cohortes de clases medias sí importa desde el punto de vista de los ingresos, lo que no está declinando. Más aún, esto se relaciona estrechamente con la composición de las nuevas cohortes de clases medias antes expuesta. Los ingresos relativamente mayores de la clase de servicios se vinculan al aumento del porcentaje de pertenecientes a esta clase entre las cohortes más recientes. En contraste, los integrantes de la clase de rutinas no-manuales no obtienen ingresos similares a la clase de servicios ni aumentan en las nuevas cohortes. Así, en estas nuevas cohortes de clases medias se aprecia una visible correspondencia de sus ingresos medios — según el modelo de regresión lineal— con la creciente o decreciente pertenencia a la respectiva clase.

En síntesis, los resultados aquí señalados muestran que las nuevas cohortes de clases medias no constituyen una mera clasificación ocupacional, sino que al menos desde el punto de vista de los ingresos, comparten condiciones similares que las distinguen de otras clases y de las viejas cohortes de clase media. Además, en el interior de las nuevas cohortes de clase media se aprecian diferencias de ingreso resultantes de la pertenencia a uno u otro estrato de clase media. Más aún, en las nuevas cohortes de la clase de servicios, que ejerce una fuerza motriz en las clases medias, la pertenencia de clase está teniendo una importante e incluso creciente incidencia relativa en los ingresos en los años recientes. En este sentido, no se verificaría un acercamiento a un tipo de sociedad crecientemente "meritocrática", en que las oportunidades y los ingresos se determinen sobre todo por el nivel educacional alcanzado. 


\section{$\mathrm{V}$}

\section{Conclusiones}

Los procesos económico-sociales experimentados por la sociedad chilena durante las últimas dos décadas han dado lugar a un cambio en las clases medias. El análisis de cohortes que se ha efectuado en este estudio evidencia el surgimiento de una nueva clase media entre quienes nacieron a partir de 1970 y se incorporaron plenamente a la actividad laboral durante el período de crecimiento económico sostenido de las décadas de 1990 y 2000. Esta dinámica y el quiebre experimentado por quienes nacieron a partir de 1970 constituye un aporte interesante de la aplicación de la metodología del análisis de cohortes al estudio de las clases medias.

Además, este análisis de cohortes permite clarificar que el proceso ocurrido no solo se reduce a una clase media emergente desde la pobreza, sino que debe ser descrito de un modo más amplio abarcando a las clases medias. Se puede concluir que la clase de servicios ocupa un lugar preeminente dentro de las clases medias, específicamente en las nuevas clases medias, lo que permite comprender mejor la clase media actual. En efecto, teniendo como referencia la ocupación total en el país, las nuevas cohortes de clases medias han experimentado un mayor ascenso en su participación en la clase de servicios que en otros estratos de clase media $\mathrm{y}$, en este sentido, esta clase es la más dinámica en las nuevas clases medias. Sus ingresos derivados de esta pertenencia de clase son relativamente más elevados en comparación con las viejas cohortes de la misma clase y respecto de otros estratos de clases medias. En otras palabras, la clase media en la sociedad chilena contemporánea es primordialmente la nueva clase de los profesionales y técnicos, en su mayor parte con educación superior, contratados en empresas o instituciones. Esto define un antes y un después en la clase media chilena, desde el punto de vista de las cohortes que la integran.

La pertenencia a las nuevas clases medias incide en los ingresos de las personas, junto con otros factores como el nivel educacional, lo que contribuye a entender mejor algunas de las causas de la desigualdad de ingresos en la sociedad chilena. Además, hay un efecto social, político y cultural provocado por el surgimiento de una generación de nuevas clases medias, que posiblemente está comenzando a incidir en orientaciones centrales de la sociedad actual y de actores como los movimientos sociales.

Así, el método de análisis de cohortes aplicado en este artículo ofrece una base sistemática con respecto a una posible trayectoria actual y futura de las clases medias. Se puede postular que las nuevas clases medias comparten una situación generacional que las induce y las impulsará a buscar oportunidades más favorables enfrentando las desigualdades sociales erigidas por otros. Esto acota el problema de las solidaridades colectivas planteado al inicio de este artículo, aunque no lo resuelve, pues la situación compartida por las nuevas clases medias es solo el comienzo del problema generacional —en el sentido de Mannheim (1952)—, que consiste en la presencia o ausencia de un vínculo en torno de un destino común. En especial, está por definirse si asume una voluntad mesocrática, entendida como el gobierno de las clases medias, lo que se encuentra fuera del foco de este artículo.

Bibliografía

Autor, D., L. Katz y A. Krueger (2008), "Trends in U.S. wage inequality: revising the revisionists", The Review of Economics and Statistics, vol. 90, No 2, Cambridge, Massachusetts, MIT Press, mayo.

Banerjee, A.V. y E. Duflo (2008), "What is middle class about the middle classes around the world?", Journal of Economic Perspectives, vol. 22, $\mathrm{N}^{\mathrm{o}}$ 2, Nashville, Tennessee, American Economic Association.

Barozet, E. (2006), "El valor histórico del pituto: Clase media, integración y diferenciación social en Chile", Revista de sociología, $\mathrm{N}^{\circ} 20$, Santiago de Chile, Universidad de Chile.

Becker, G. (1975), Human Capital: A Theoretical and Empirical Analysis with Special Reference to Education, Chicago, University of Chicago Press.
Bourdieu, P. (1987), La distinción: Criterios y bases sociales del gusto, Madrid, Taurus.

Chauvel, L. (2002), Le destin des générations, structure sociale et cohortes en France au XXe siècle, París, Presses Universitaires de France (PUF).

Erikson, R. y J.H. Goldthorpe (1993), The Constant Flux: A Study of Class Mobility in Industrial Societies, Oxford, Oxford University Press.

Espinoza, V. y E. Barozet (2009), “¿De qué hablamos cuando decimos 'clase media'? Perspectivas sobre el caso chileno", En foco, $\mathrm{N}^{\circ}$ 142, Santiago de Chile, Expansiva.

Franco, R., A. León y R. Atria (2007), "Estratificación y movilidad social en América Latina. Una agenda de trabajo", Estratificación 
y movilidad social en América Latina. Transformaciones estructurales de un cuarto de siglo, R. Franco, A. León y R. Atria (eds.), Santiago de Chile, LOM Ediciones.

Franco, R., M. Hopenhayn y A. León (2011), "Crece y cambia la clase media en América Latina: Una puesta al día”, Revista de la CEPAL, No 103 (LC/G.2487-P), Santiago de Chile, abril. (2010), "Las clases medias en América Latina: Historias cruzadas y miradas diversas", Las clases medias en América Latina: Retrospectiva y nuevas tendencias, R. Franco, M. Hopenhayn y A. León (coords.), México, D.F., CEPAL-Siglo XXI.

Garretón, M.A. (2007), The Socio-political Matrix and Economic Development in Chile, Manchester, University of Manchester, octubre.

Glenn, N. (2005), Cohort Analysis, Thousand Oaks, California, Sage Publications.

Kreckel, R. (2006), “On national and global 'middle classes”, documento preparado para el Indian-German Workshop, Universidad de Munich, 7 y 8 de septiembre.

Leiulfsrud, H., I. Bison y H. Jensberg (2005), Social Class in Europe: European Social Survey 2002/3, Trondheim, Norwegian University of Science and Technology (NTNU).

León, A. y J. Martínez (2001), "La estratificación social chilena hacia fines del siglo XX", serie Políticas sociales, $\mathrm{N}^{\circ} 52$ (LC/L.1584-P), Santiago de Chile, Comisión Económica para América Latina y el Caribe (CEPAL), agosto. Publicación de las Naciones Unidas, $\mathrm{N}^{\circ}$ de venta: S.01.II.G.127.

Mannheim, K. (1952), "The problem of generations", Essays on the Sociology of Knowledge, K. Mannheim, Londres, Routledge \& Kegan Paul. Publicado originalmente en 1928.
Marx, K. y F. Engels (1989), Contribución a la crítica de la economía política, Moscú, Editorial Progreso. Publicado originalmente en 1859.

Mincer, J. (1974), Schooling, Experience and Earnings, Nueva York, National Bureau of Economic Research.

OCDE (Organización de Cooperación y Desarrollo Económicos) (2011), Society at a Glance 2011: OECD Social Indicators, OECD Publishing [en línea] http://dx.doi.org/10.1787/soc_glance-2011-en.

(2010), Latin American Economic Outlook 2011. How Middleclass is Latin America? [en línea] http://www.latameconomy.org/ fileadmin/uploads/laeo/Documents/E-book_LEo2011-EN_entier.pdf

Ravallion, M. (2009), "The developing world's bulging (but vulnerable) 'middle class",, Policy Research Working Paper, No 4816, Washington, D.C., Banco Mundial.

Ryder, N. (1965), "The cohort as a concept in the study of social change", American Sociological Review, vol. 30, $\mathrm{N}^{\circ}$ 6, Washington, D.C., American Sociological Association, diciembre.

Torche, F. y G. Wormald (2004), "Estratificación y movilidad social en Chile: Entre la adscripción y el logro", serie Políticas sociales, No 98 (LC/L.2209-P), Santiago de Chile, Comisión Económica para América Latina y el Caribe (CEPAL). Publicación de las Naciones Unidas, $\mathrm{N}^{\circ}$ de venta: S.04.II.G.132.

Sapelli, C. (2011a), "A cohort analysis of the income distribution in Chile", Estudios de economía, vol. 38, № 1, Santiago de Chile, Universidad de Chile, junio.

(2011b), Chile: ¿Más equitativo? Una mirada distinta a la distribución del ingreso, la movilidad social y la pobreza en Chile, Santiago de Chile, Ediciones Universidad Católica de Chile.

Weber, M. (1964), Economía y sociedad, México, D.F., Fondo de Cultura Económica. Publicado originalmente en 1922. 